\title{
Intelligent Reflecting Surface Enhanced Secure Transmission Against Both Jamming and Eavesdropping Attacks
}

\author{
Yifu Sun, Kang An, Junshan Luo, Yonggang Zhu, Gan Zheng, Fellow, IEEE, and Symeon Chatzinotas, Senior \\ Member, IEEE
}

\begin{abstract}
Both the jammer and the eavesdropper pose severe threat to wireless communications due to the broadcast nature of wireless channels. In this paper, an intelligent reflecting surface (IRS) assisted secure communication system is considered, where a base station (BS) wishes to reliably convey information to a user, in the presence of both a jammer and an eavesdropper whose channel state information (CSI) is not perfectly known. Specifically, we aim to maximize the system achievable rate by jointly designing the BS's transmit beamforming and the IRS's reflect beamforming with imperfect CSI, while limiting the information leakage to the potential eavesdropper. Due to the non-convexity and intractability of the original problem induced by the CSI uncertainty, we utilize the auxiliary variables and General Sign-Definiteness transformation to convert the original optimization problem into a tractable convex optimization problem, and then obtain the high-quality optimal solution by using the successive convex approximation and penalty convex concave procedure. Numerical simulations demonstrate the superiority of our proposed optimization algorithm compared with existing approaches, and also reveal the impact of key parameters on the achievable system performance.
\end{abstract}

Index Terms-Intelligent reflecting surface, anti-jamming, physical-layer security, robust beamforming optimization, imperfect channel state information (CSI).

\section{INTRODUCTION}

$\mathbf{T}$ HE advancement of the next generation wireless communication explosively increases the demands on data transmission. However, due to the broadcast nature of wireless channels, the associated security vulnerabilities and threats have raised growing concerns, including both the passive eavesdropping for data interception and the active jamming for disrupting legitimate transmissions [1]. Various technologies have been proposed to enhance wireless security against the jamming and eavesdropping attacks. Frequency hopping [2] and power control [3] are extensively adopted to address the jamming attacks. However, frequency hopping consumes extra

This work is supported by the NSFC under Grants U19B214 and 61901502, partly by the Foundation strengthening Plan Area Fund under Grants 2019JCJQ-JJ-212 and 2019-JCJQ-JJ226, partly funded by FNR RISOTTI. (Corresponding author: Yonggang Zhu and Kang An)

Y. Sun, K. An, J. Luo, and Y. Zhu are with National University of Defense Technology, China (Email: sunyifu.nudt@nudt.edu.cn; ankang89@nudt.edu.cn; ljsnudt@ foxmail.com; zhumaka1982@163.com).

G. Zheng is with the Wolfson School of Mechanical, Electrical and Manufacturing Engineering, Loughborough University, Loughborough LE11 3TU, U.K. (e-mail: g.zheng@ @lboro.ac.uk).

S. Chatzinotas is with Interdisciplinary Centre for Security, Reliability and Trust, University of Luxembourg, Luxembourg, L-1855, Luxembourg (e-mail: symeon.chatzinotas@uni.lu). spectrum resources, and power control is not suitable for the case of large jamming power. In terms of the eavesdropping, the existing literatures usually applied cooperative relaying [4] and artificial noise-aided beamforming [5] schemes. Nevertheless, cooperative relaying and transmitting artificial noise consume additional power.

To overcome these shortcomings of existing approaches, a new paradigm, called intelligent reflecting surface (IRS), has been recently proposed to enhance both secrecy performance and spectrum efficiency [6]-[13]. An IRS is comprised of many passive low-cost reflecting elements, where each units can adaptively adjust its phase and/or amplitude to reconfigure the wireless propagation environment, thus boosting and/or suppressing the received signals at the users [6]-[8]. In [8], by leveraging the passive IRS, the authors jointly optimized the active beamforming at BS and the passive beamforming at IRS to improve the coverage of wireless network. Aiming to maximize the achievable secrecy rate, the authors in [9] and [10] used IRS to protect secure transmission form eavesdropping attacks. The works in [11] further studied the IRS-assisted secure beamforming and artificial noise scheme to miximized the the secrecy rate in the multiple-input multiple-output (MIMO) system. Considering the channel state information (CSI) is not perfectly known at the base station (BS), a transmit power minimization problem is formulated in [12] for antieavesdropping with imperfect CSI. Despite the above works focusing on the anti-eavesdropping scenarios, a prior work in [13] first used IRS for anti-jamming communications with the aim of maximizing the system achievable rate under the minimum SINR requirement and perfect CSI assumption. To the best of our knowledge, no exiting work has considered the utilization and associated design of IRS-assisted secure transmission against both jamming and eavesdropping attacks with imperfect CSI.

In this paper, we propose an IRS-enhanced secure communication system for protecting the wireless transmission from both jamming and eavesdropping attacks, where the third-party node's CSI is not perfectly known at the BS. The contributions of this paper are summarized as follows:

- A generalized framework of IRS-assisted secure transmission against both jammer and eavesdropper is first proposed. In addition, we consider the robust beamforming design with imperfect CSI. Specifically, taking the bounded jammer's and eavesdropper's CSI into account, the achievable system rate is maximized by 
jointly optimizing the active transmit beamforming at BS and the passive reflecting beamforming at IRS, while the information leakage to the potential eavesdropper is constrained.

- Owing to the non-convexity and intractability of optimization problem, we firstly transform the non-convex objective function into convex one by adding a auxiliary variable, and subsequently, the General Sign-Definiteness transformation is applied to address the CSI uncertainty. As such, the successive convex approximation (SCA) and penalty convex concave procedure $(\mathrm{P}-\mathrm{CCP})$ are proposed to solve the optimization problem.

- Numerical results demonstrate the effectiveness and superiority of the proposed scheme, through comparison to the existing approach, non-IRS scheme as well as heuristic beamforming design.

\section{System Model AND PROBlem Formulation}

\section{A. System Model}

As depicted in Fig.1, a BS with $M$ antennas wishes to reliably communicate with a single-antenna user with the aid of a IRS, in the presence of a jammer with $L$ antennas and a single-antenna eavesdropper. It is assumed that the IRS is equipped with $N$ reflecting elements and controlled by a microchip which coordinates the BS and IRS for the channel acquisition and data transmission [7]. The channel coefficients of the BS-user link, the BS-eavesdropper link, the jammer-user link, the BS-IRS link, the jammer-IRS link, the IRS-user link, and the IRS-eavesdropper link are denoted by $\mathbf{h}_{\mathrm{BU}} \in \mathbb{C}^{1 \times M}, \mathbf{h}_{\mathrm{BE}} \in \mathbb{C}^{1 \times M}, \mathbf{h}_{\mathrm{JU}} \in \mathbb{C}^{1 \times L}, \mathbf{G}_{\mathrm{BI}} \in \mathbb{C}^{N \times M}$, $\mathbf{G}_{\mathrm{JI}} \in \mathbb{C}^{N \times L}, \mathbf{h}_{\mathrm{IU}} \in \mathbb{C}^{1 \times N}$, and $\mathbf{h}_{\mathrm{IE}} \in \mathbb{C}^{1 \times N}$, respectively. We assume that the CSI of legitimate channels $\left(\mathbf{h}_{\mathrm{BU}}, \mathbf{G}_{\mathrm{BI}}\right.$, and $\mathbf{h}_{\mathrm{IU}}$ ) can be accurately obtained due to the slow-varying property of channels [14]. In addition, due to the lack of cooperation between the BS and the third-party nodes, the CSI of illegitimate channels are challenging to be acquired. To account for this effect, the bounded CSI model is adopted to characterize the CSI uncertainty of illegitimate channels. For subsequent analysis, we denote by $\mathbf{H}_{\mathrm{BU}}=\operatorname{diag}\left(\mathbf{h}_{\mathrm{IU}}\right) \mathbf{G}_{\mathrm{BI}}$, $\mathbf{H}_{\mathrm{JU}}=\operatorname{diag}\left(\mathbf{h}_{\mathrm{IU}}\right) \mathbf{G}_{\mathrm{JI}}$, and $\mathbf{H}_{\mathrm{BE}}=\operatorname{diag}\left(\mathbf{h}_{\mathrm{IE}}\right) \mathbf{G}_{\mathrm{BI}}$ as the cascaded channels of BS-IRS-user link, jammer-IRS-user link, and BS-IRS-eavesdropper link, respectively. As such, the bounded CSI of illegitimate channels can be expressed as

$$
\begin{aligned}
& \mathbf{h}_{i}=\widehat{\mathbf{h}}_{i}+\Delta \mathbf{h}_{i},\left\|\Delta \mathbf{h}_{i}\right\| \leq \xi_{\mathrm{h}, i}, i \in(\mathrm{JU}, \mathrm{BE}) \\
& \mathbf{H}_{i}=\widehat{\mathbf{H}}_{i}+\Delta \mathbf{H}_{i},\left\|\Delta \mathbf{H}_{i}\right\| \leq \xi_{\mathrm{H}, i},
\end{aligned}
$$

where $\widehat{\mathbf{h}}_{i}$ and $\widehat{\mathbf{H}}_{i}$ denote the estimated CSI known at BS, $\Delta \mathbf{h}_{i}$ and $\Delta \mathbf{H}_{i}$ are unknown CSI error. and $\xi_{\mathrm{H}, i}, \xi_{\mathrm{h}, i}$ represent the CSI uncertainty level.

The desired signal that BS intended for the user is $s_{\mathrm{T}}$ with zero mean and unit variance, which is weighted by the transmit beamforming vector $\mathbf{w}_{\mathrm{T}} \in \mathbb{C}^{M \times 1}$. Thus, the overall transmitted signal can be expressed as $\mathbf{x}=\mathbf{w}_{\mathrm{T}} s_{\mathrm{T}}$, where $\mathrm{E}\left[\left|s_{\mathrm{T}}\right|^{2}\right]=1$. Here, $\mathrm{E}[\cdot]$ and $|\cdot|$ denote the expectation and the modulus of a complex number or matrix, respectively. Owing to the fact that the BS's energy supply is limited, the

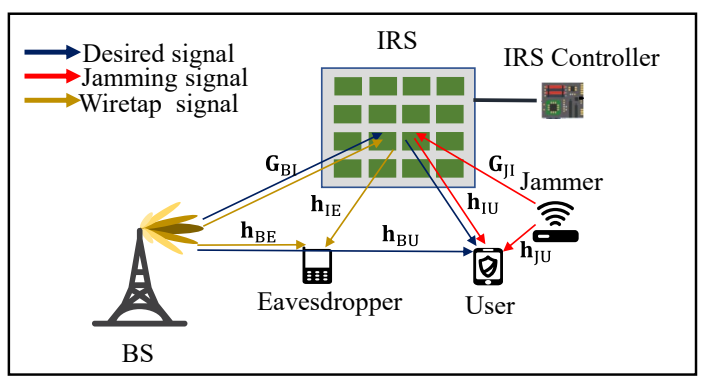

Fig. 1: System model.

transmit power vector satisfies $\left\|\mathbf{w}_{\mathrm{T}}\right\|^{2} \leq P_{\max }$, where $\|\cdot\|$ denotes the Euclidean 2-norm, and $P_{\max }$ is the maximum BS's transmit power. The multi-antenn jammer sends the jamming signal $\mathbf{w}_{\mathrm{J}} s_{\mathrm{J}} \in \mathbb{C}^{L \times 1}$ to the user for interrupting the communication, where $\mathrm{E}\left[\left|s_{\mathrm{J}}\right|^{2}\right]=1$. Each IRS element reflects a superimposed signal to both user and eavesdropper. We denote the reflection coefficient matrix of IRS by $\mathbf{v}=\left(\mathrm{v}_{1}, \ldots, \mathrm{v}_{N}\right)^{T}$, where $\mathrm{v}_{i}=e^{j \theta_{i}}, \theta_{i} \in[0,2 \pi]$ and $\left|\mathrm{v}_{i}\right|=1, \forall i$. Due to the severe path loss, the signals reflected by the IRS two or more times can be ignored. Additionally, considering the potential cooperation between the jammer and the eavesdropper, the jamming signal recieved by the eavesdropper can be eliminated ${ }^{1}$. Hence, the received signals at the user and the eavesdropper are respectively given by

$$
\begin{aligned}
& y_{\mathrm{U}}=\widetilde{\mathbf{h}}_{\mathrm{BU}} \mathbf{w}_{\mathrm{T}} s_{\mathrm{T}}+\widetilde{\mathbf{h}}_{\mathrm{JU}} \mathbf{w}_{\mathrm{J}} s_{\mathrm{J}}+n_{\mathrm{U}}, n_{\mathrm{U}} \sim \mathcal{C N}\left(0, \sigma_{\mathrm{U}}^{2}\right) \\
& y_{\mathrm{E}}=\widetilde{\mathbf{h}}_{\mathrm{BE}} \mathbf{w}_{\mathrm{T}} s_{\mathrm{T}}+n_{\mathrm{E}}, n_{\mathrm{E}} \sim \mathcal{C N}\left(0, \sigma_{\mathrm{E}}^{2}\right)
\end{aligned}
$$

where $\widetilde{\mathbf{h}}_{m}=\mathbf{h}_{m}+\mathbf{v}^{H} \mathbf{H}_{m},, m \in(\mathrm{BU}, \mathrm{JU}, \mathrm{BE})$, and $\mathcal{C N}$ denotes the distribution of a circularly symmetric complex Gaussian random vector. Accordingly, the system achievable rate and the secrecy rate can be respectively expressed as

$$
\begin{aligned}
& R_{\mathrm{U}}\left(\mathbf{w}_{\mathrm{T}}, \mathbf{v}\right)=\log _{2}\left(1+\frac{\left|\widetilde{\mathbf{h}}_{\mathrm{BU}} \mathbf{w}_{\mathrm{T}}\right|^{2}}{\left|\widetilde{\mathbf{h}}_{\mathrm{JU}} \mathbf{w}_{\mathrm{J}}\right|^{2}+\sigma_{\mathrm{U}}^{2}}\right), \\
& C_{\mathrm{sec}}\left(\mathbf{w}_{\mathrm{T}}, \mathbf{v}\right)=\left[R_{\mathrm{U}}\left(\mathbf{w}_{\mathrm{T}}, \mathbf{v}\right)-R_{\mathrm{E}}\left(\mathbf{w}_{\mathrm{T}}, \mathbf{v}\right)\right]^{+},
\end{aligned}
$$

where $R_{\mathrm{E}}\left(\mathbf{w}_{\mathrm{T}}, \mathbf{v}\right)=\log _{2}\left(1+\frac{\left|\widetilde{\mathbf{h}}_{\mathrm{BE}} \mathbf{w}_{\mathrm{T}}\right|^{2}}{\sigma_{\mathrm{E}}{ }^{2}}\right)$ and $[z]^{+}=$ $\max (z, 0)$.

\section{B. Problem Formulation}

In this paper, a worst-case robust rate maximization problem is formulated for the bounded CSI uncertainties [17]. In particular, we aim to maximize the system achievable rate by jointly designing the transmit beamforming $\mathbf{w}_{\mathrm{T}}$ at $\mathrm{BS}$

\footnotetext{
${ }^{1}$ Due to the cooperation between the jammer and the eavesdropper, the jammer can design the beam pattern which positions the eavesdropper in the null space of the jamming signal by using the multi-antenna technique [15]. In addition, the work in [16] has summarized some signal processing methods to address the interference probelm, where the receivers can use Code Division Multiple Access(CDMA), core decoding, and detection technique to eliminate the interference. Therefore, it is reasonable to assume that the jamming signal recieved by the eavesdropper can be eliminated.
} 
and the refecting beamforming vector $\mathbf{v}$ at the IRS against both jamming and eavesdropping attacks, while keeping the information leakage to the eavesdropper below a target. Thus, the corresponding problem ${ }^{2}$ can be formulated as

$$
\begin{aligned}
\mathcal{F}: & \max _{\mathbf{w}_{\mathrm{T}}, \mathbf{v}} \min _{\Delta \mathbf{h}_{\mathrm{JU}}, \Delta \mathbf{H}_{\mathrm{JU}}} R_{\mathrm{U}}\left(\mathbf{w}_{\mathrm{T}}, \mathbf{v}\right), \\
\text { s.t. } \mathrm{C} 1: \max _{\Delta \mathbf{h}_{\mathrm{BE}} \Delta \mathbf{H}_{\mathrm{BE}}} R_{\mathrm{E}}\left(\mathbf{w}_{\mathrm{T}}, \mathbf{v}\right) \leq \tau, & \\
& \mathrm{C} 2:\left\|\mathbf{w}_{\mathrm{T}}\right\|^{2} \leq P_{\max }, \mathrm{C} 3:\left|\mathrm{v}_{i}\right|=1, \forall i,
\end{aligned}
$$

where $\tau$ is the target secrecy rate. Note that the optimization problem $\mathcal{F}$ is non-convex and we cannot solve it directly, due to the coupled variables $\mathbf{w}_{\mathrm{T}}$ and $\mathbf{v}$ in both the objective function and the constraints. In addition, the CSI uncertainty is considered in the problem, which leads to infinitely nonconvex constraints in the objective function and $\mathrm{C} 1$, which forms another challenge for solving the problem $\mathcal{F}$. Thus, we propose an alternative algorithm (AO) to solve the problem in the following section.

\section{System Achievable Rate Maximization}

In this section, we divide problem $\mathcal{F}$ into two subproblems, i.e., the robust secure beamforming and phase shift design, and then $\mathbf{w}_{\mathrm{T}}$ and $\mathbf{v}$ can be obtained in an iterative manner.

\section{A. Robust Secure Beamforming Design}

Given the phase shift $\mathbf{v}$, we try to achieve the robust secure beamforming $\mathbf{w}_{\mathrm{T}}$ under imperfect CSI in this subsection. Since the term $\left|\widetilde{\mathbf{h}}_{\mathrm{JU}} \mathbf{w}_{\mathrm{J}}\right|^{2}$ in problem $\mathcal{F}$ does not involve $\mathbf{w}_{\mathrm{T}}$, we can regard it as a fixed jamming power $J$ in the subproblem. Hence, the CSI uncertainties in the objective function can be ignored due to the abovementioned operation, and then the problem $\mathcal{F}$ can be reformulated as

$$
\mathcal{Q}_{1}^{w}: \max _{\mathbf{w}_{\mathrm{T}}}\left|\widetilde{\mathbf{h}}_{\mathrm{BU}} \mathbf{w}_{\mathrm{T}}\right|^{2} \text { s.t. } \mathrm{C} 1, \mathrm{C} 2 .
$$

Indeed, problem $\mathcal{Q}_{1}^{w}$ remains non-convex as its objective function and constraints are non-convex. To address this difficulties, we first equivalently convert the infinite non-convex constraint $\mathrm{C} 1$ into a tractable form by utilizing the following proposition.

Proposition 1: Constraint $\mathrm{C} 1$ has the following equivalent tractable form $\overline{\mathrm{C} 1}$, which is formulated as

$$
\left[\begin{array}{cccc}
\widehat{a}_{\mathrm{BE}} & \widehat{\mathbf{A}}_{\mathrm{BE}} & \mathbf{0}_{1 \times M} & \mathbf{0}_{1 \times M} \\
\widehat{\mathbf{A}}_{\mathrm{BE}}^{H} & 1-u_{2} & \xi_{\mathrm{H}, \mathrm{BE}} \mathbf{w}_{\mathrm{T}}^{H} & \xi_{\mathrm{h}, \mathrm{BE}} \mathbf{W}_{\mathrm{T}}^{H} \\
\mathbf{0}_{M \times 1} & \xi_{\mathrm{H}, \mathrm{BE}} \mathbf{W}_{\mathrm{T}} & u_{1} \mathbf{I} & \mathbf{0}_{M \times M} \\
\mathbf{0}_{M \times 1} & \xi_{\mathrm{h}, \mathrm{BE}} \mathbf{W}_{\mathrm{T}} & \mathbf{0}_{M \times M} & u_{2} \mathbf{I}
\end{array}\right] \succeq 0,
$$

where $\widehat{\mathbf{A}}_{\mathrm{BE}}=\left(\widehat{\mathbf{h}}_{\mathrm{BE}}+\mathbf{v}^{H} \widehat{\mathbf{H}}_{\mathrm{BE}}\right) \mathbf{w}_{\mathrm{T}}, \widehat{a}_{\mathrm{BE}}=\sigma_{\mathrm{E}}^{2}\left(2^{\tau}-1\right)-u_{1} N-$ $u_{2}$, and $u_{1}, u_{2} \geq 0$ are slack variables.

Proof: By dropping the log function and adopting Schurs complement [18] in $\mathrm{C} 1$, the constraint $\mathrm{C} 1$ can be equivalently transformed to

$$
\left[\begin{array}{cc}
\sigma_{\mathrm{E}}^{2}\left(2^{\tau}-1\right) & \widetilde{\mathbf{h}}_{\mathrm{BE}} \mathbf{w}_{\mathrm{T}} \\
\mathbf{w}_{\mathrm{T}}^{H} \widetilde{\mathbf{h}}_{\mathrm{BE}}^{H} & 1
\end{array}\right] \succeq 0 .
$$

\footnotetext{
${ }^{2}$ According to [10], the constraint $\mathrm{C} 1$ guarantees the system secrecy rate is bounded from $C_{\mathrm{sec}}\left(\mathbf{w}_{\mathrm{T}}, \mathbf{v}\right) \geq R_{\mathrm{U}}\left(\mathbf{w}_{\mathrm{T}}, \mathbf{v}\right)-\tau$.
}

Then, substituting $\mathbf{h}_{\mathrm{BE}}=\widehat{\mathbf{h}}_{\mathrm{BE}}+\Delta \mathbf{h}_{\mathrm{BE}}, \mathbf{H}_{\mathrm{BE}}=\widehat{\mathbf{H}}_{\mathrm{BE}}+\Delta \mathbf{H}_{\mathrm{BE}}$ into (10) and after some mathematical transformations, we can obtain that

$$
\begin{aligned}
& {\left[\begin{array}{cc}
\sigma_{\mathrm{E}}^{2}\left(2^{\tau}-1\right) & \left(\widehat{\mathbf{h}}_{\mathrm{BE}}+\mathbf{v}^{H} \widehat{\mathbf{H}}_{\mathrm{BE}}\right) \mathbf{w}_{\mathrm{T}} \\
\mathbf{w}_{\mathrm{T}}^{H}\left(\widehat{\mathbf{h}}_{\mathrm{BE}}^{H}+\widehat{\mathbf{H}}_{\mathrm{BE}}^{H} \mathbf{v}\right) & 1
\end{array}\right] \succeq} \\
& -\left[\begin{array}{c}
\mathbf{0}_{1 \times M} \\
\mathbf{w}_{\mathrm{T}}^{H}
\end{array}\right]\left[\Delta \mathbf{h}_{\mathrm{BE}}^{H} \mathbf{0}_{M \times 1}\right] \mathbf{I}-\mathbf{I}\left[\begin{array}{c}
\Delta \mathbf{h}_{\mathrm{BE}} \\
\mathbf{0}_{1 \times M}
\end{array}\right]\left[\mathbf{0}_{M \times 1} \mathbf{w}_{\mathrm{T}}\right]
\end{aligned}
$$

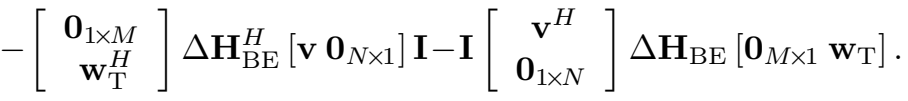

Next, we utilize General Sign-Definiteness transformation to make further manipulations, which is expressed as

Lemma 1: (General Sign-Definiteness [19]) Given matrices $\mathbf{B}=\mathbf{B}^{H}$ and $\left\{\mathbf{C}_{i}, \mathbf{D}_{i}\right\}_{i=1}^{P}$, the linear matrix inequality (LMI) $\mathbf{B} \succeq \sum_{i=1}^{P}\left(\mathbf{C}_{i}^{H} \mathbf{X}_{i} \mathbf{D}_{i}+\mathbf{D}_{i}^{H} \mathbf{X}_{i}^{H} \mathbf{C}_{i}\right), \forall i,\left\|\mathbf{X}_{i}\right\| \leq \xi_{i}$ hold only if there exists $u_{i} \geq 0, \forall i$, such that

$$
\left[\begin{array}{cccc}
\mathbf{B}-\sum_{i=1}^{P} u_{i} \mathbf{D}_{i}^{H} \mathbf{D}_{i} & -\xi_{1} \mathbf{C}_{1}^{H} & \cdots & -\xi_{P} \mathbf{C}_{P}^{H} \\
-\xi_{1} \mathbf{C}_{1} & u_{i} \mathbf{I} & \cdots & \mathbf{0} \\
\vdots & \vdots & \ddots & \vdots \\
-\xi_{P} \mathbf{C}_{P} & \mathbf{0} & \cdots & u_{P} \mathbf{I}
\end{array}\right] \succeq 0 .
$$

Proof: Please refer to [19].

In order to use lemma 1, we choose the following parameters to replace the terms in (11), as

$$
\begin{aligned}
& \mathbf{B}=\left[\begin{array}{cc}
\sigma_{\mathrm{E}}^{2}\left(2^{\tau}-1\right) & \left(\widehat{\mathbf{h}}_{\mathrm{BE}}+\mathbf{v}^{H} \widehat{\mathbf{H}}_{\mathrm{BE}}\right) \mathbf{w}_{\mathrm{T}} \\
\mathbf{w}_{\mathrm{T}}^{H}\left(\widehat{\mathbf{h}}_{\mathrm{BE}}^{H}+\widehat{\mathbf{H}}_{\mathrm{BE}}^{H} \mathbf{v}\right) & 1
\end{array}\right] \\
& \mathbf{C}_{1}=\mathbf{C}_{2}=-\left[\begin{array}{ll}
\mathbf{0}_{M \times 1} & \mathbf{w}_{\mathrm{T}}
\end{array}\right], \mathbf{D}_{1}=\left[\begin{array}{ll}
\mathbf{v} & \mathbf{0}_{N \times 1}
\end{array}\right] \mathbf{I}, \mathbf{D}_{\mathbf{2}}=\mathbf{I} \\
& \mathbf{X}_{1}=\Delta \mathbf{H}_{\mathrm{BE}}^{H}, \mathbf{X}_{2}=\left[\begin{array}{ll}
\Delta \mathbf{h}_{\mathrm{BE}}^{H} & \mathbf{0}_{M \times 1}
\end{array}\right] .
\end{aligned}
$$

Applying the lemma 1 , by introducing slack variables $u_{1}, u_{2}$, and combing $\left\|\Delta \mathbf{h}_{\mathrm{BE}}\right\| \leq \xi_{\mathrm{h}, \mathrm{BE}},\left\|\Delta \mathbf{H}_{\mathrm{BE}}\right\|_{F} \leq \xi_{\mathrm{H}, \mathrm{BE}}$, (11) can be transformed into a LMI $\overline{\mathrm{C} 1}$.

Hence, the proof is completed.

Note that the objective function in the problem $\mathcal{Q}_{1}^{w}$ is nonconvex respect to $\mathbf{w}_{\mathrm{T}}$. Nevertheless, we can approximate it via SCA. By utilizing the first-order Taylor inequality, i.e., for any complex scalar variable $x$ and $x^{(n)}$,

$$
|x|^{2} \geq 2 \operatorname{Re}\left\{x^{(n), *} x\right\}-x^{(n), *} x^{(n)},
$$

we can obtain $\left|\widetilde{\mathbf{h}}_{\mathrm{BU}} \mathbf{w}_{\mathrm{T}}\right|^{2} \geq 2 \operatorname{Re}\left\{\widetilde{\mathbf{h}}_{\mathrm{BU}} \mathbf{w}_{\mathrm{T}}^{(n)} \mathbf{w}_{\mathrm{T}}^{H} \widetilde{\mathbf{h}}_{\mathrm{BU}}^{H}\right\}-$ $\widetilde{\mathbf{h}}_{\mathrm{BU}} \mathbf{w}_{\mathrm{T}}^{(n)} \mathbf{w}_{\mathrm{T}}^{(n), H} \widetilde{\mathbf{h}}_{\mathrm{BU}}^{H}$, where $\mathbf{w}_{\mathrm{T}}^{(\mathbf{n})}$ is optimal solution obtained at iteration $n$. Here, the superscript $*$ and $T$ represent the conjugate and transpose, respectively. Thus, after dropping the the constant term, the problem $\mathcal{Q}_{1}^{w}$ can be recast as

$$
\mathcal{Q}^{w}: \max _{\mathbf{w}_{\mathrm{T}},\left\{u_{1}, u_{2}\right\} \geq 0} \operatorname{Re}\left\{\mathbf{w}_{\mathrm{T}}^{H} \widetilde{\mathbf{h}}_{\mathrm{BU}}^{H} \widetilde{\mathbf{h}}_{\mathrm{BU}} \mathbf{w}_{\mathrm{T}}^{(n)}\right\} \text { s.t. } \overline{\mathrm{C} 1}, \mathrm{C} 2 .
$$

Obviously, the convex problem $\mathcal{Q}^{w}$ can be solved by using the CVX tool [20]. Therefore, a first-order optimal solution of $\mathbf{w}_{\mathrm{T}}$ given $\mathbf{v}$ can be achieved by utilizing SCA to solve the problem $\mathcal{Q}^{w}$ until convergence. 


\section{B. Phase Shift Design}

Given the transmit beamforming $\mathbf{w}_{\mathrm{T}}$, recall the transformed constraint $\overline{\mathrm{C} 1}$, and then the phase shift design problem can be expressed as

$$
\mathcal{Q}_{1}^{v}: \max _{\mathbf{v},\left\{u_{1}, u_{2}\right\} \geq 0} \min _{\Delta \mathbf{h}_{\mathrm{JU}}, \Delta \mathbf{H}_{\mathrm{JU}}} \frac{\left|\widetilde{\mathbf{h}}_{\mathrm{BU}} \mathbf{w}_{\mathrm{T}}\right|^{2}}{\left|\widetilde{\mathbf{h}}_{\mathrm{JU}} \mathbf{w}_{\mathrm{J}}\right|^{2}+\sigma_{\mathrm{U}}^{2}} \text {, s.t. } \overline{\mathrm{C} 1}, \mathrm{C} 3 .
$$

However, problem $\mathcal{Q}_{1}^{v}$ is still non-convex due to $\mathbf{v}$ and the infinite concave bounded estimation error objective function. To solve this problem, we first convert the objective function into a more tractable form by adding an auxiliary variable $\eta \geq 0$, and then problem $\mathcal{Q}_{1}^{v}$ can be reformulated as

$$
\begin{aligned}
& \mathcal{Q}_{2}^{v}: \max _{\mathbf{v},\left\{u_{1}, u_{2}, \eta\right\} \geq 0} \frac{\left|\widetilde{\mathbf{h}}_{\mathrm{BU}} \mathbf{w}_{\mathrm{T}}\right|^{2}}{\eta+\sigma_{\mathrm{U}}^{2}} \\
& \text { s.t. } \overline{\mathrm{C}} 1, \mathrm{C} 3, \mathrm{C} 4:\left|\widetilde{\mathbf{h}}_{\mathrm{JU}} \mathbf{w}_{\mathrm{J}}\right|^{2} \leq \eta .
\end{aligned}
$$

Similar to the proposition 1, the constraint $\mathrm{C} 4$ can be equivalently transformed to $\overline{\mathrm{C}} 4$, which is given by

$\left[\begin{array}{cccc}\widehat{a}_{\mathrm{JU}} & \widehat{\mathbf{A}}_{\mathrm{JU}} & \mathbf{0}_{1 \times L} & \mathbf{0}_{1 \times L} \\ \widehat{\mathbf{A}}_{\mathrm{JU}}^{H} & 1-p_{2} & \xi_{\mathrm{H}, \mathrm{JU}} \mathbf{w}_{\mathrm{J}}^{H} & \xi_{\mathrm{h}, \mathrm{JU}} \mathbf{w}_{\mathrm{J}}^{H} \\ \mathbf{0}_{L \times 1} & \xi_{\mathrm{H}, \mathrm{JU}} \mathbf{w}_{\mathrm{J}} & p_{1} \mathbf{I} & \mathbf{0}_{L \times L} \\ \mathbf{0}_{L \times 1} & \xi_{\mathrm{h}, \mathrm{JU}} \mathbf{w}_{\mathrm{J}} & \mathbf{0}_{L \times L} & p_{2} \mathbf{I}\end{array}\right] \succeq 0$,

where $\widehat{\mathbf{A}}_{\mathrm{JU}}=\left(\widehat{\mathbf{h}}_{\mathrm{JU}}+\mathbf{v}^{H} \widehat{\mathbf{H}}_{\mathrm{JU}}\right) \mathbf{w}_{\mathrm{J}}, \widehat{a}_{\mathrm{JU}}=\eta-p_{1} N-p_{2}$, and $p_{1}, p_{2} \geq 0$ are slack variables.

Proof: Please refer to the proof of Proposition 1.

Therefore, by using the aforementioned manipulation, problem $\mathcal{Q}_{2}^{v}$ can be equivalently transformed to

$$
\mathcal{Q}_{3}^{v}: \max _{\mathbf{v},\left\{p_{1}, p_{2}, p_{3}, p_{4}, u_{1}, u_{2}, \eta\right\} \geq 0} \frac{\left|\widetilde{\mathbf{h}}_{\mathrm{BU}} \mathbf{w}_{\mathrm{T}}\right|^{2}}{\eta+\sigma_{\mathrm{U}}^{2}} \text { s.t. } \overline{\mathrm{C} 1}, \mathrm{C} 3, \overline{\mathrm{C} 4} \text {. }
$$

Note that in the problem $\mathcal{Q}_{3}^{v}$, the system achievable rate is determined by $\mathbf{v}$ and $\eta$, which cannot be solved simultaneously. Thus, in this subsection, we first achieve a proper region of $\eta$ numerically, and then utilize the SCA and P-CCP to optimize $\mathbf{v}$ with fixed $\eta$. Finally, the optimal $\eta^{o p t}$ can be obtained by using sampling method in its region [17].

Specifically, in this paper, we define that the achievable rate must satisfy $R_{\mathrm{U}}\left(\mathbf{w}_{\mathrm{T}}, \mathbf{v}\right) \geq 1 \mathrm{bps} / \mathrm{Hz}$, and thus one obtains that

$$
\begin{aligned}
& \eta+\sigma_{\mathrm{U}}^{2} \leq\left|\widetilde{\mathbf{h}}_{\mathrm{BU}} \mathbf{w}_{\mathrm{T}}\right|^{2}=\left\|\mathbf{w}_{\mathrm{T}}\right\|^{2}\left\|\mathbf{h}_{\mathrm{BU}}+\mathbf{v}^{H} \mathbf{H}_{\mathrm{BU}}\right\|^{2} \\
& =\left\|\mathbf{w}_{\mathrm{T}}\right\|^{2}\left(\mathbf{h}_{\mathrm{BU}} \mathbf{h}_{\mathrm{BU}}^{H}+2 \operatorname{Re}\left\{\mathbf{v}^{H} \mathbf{H}_{\mathrm{BU}} \mathbf{h}_{\mathrm{BU}}^{H}\right\}+\mathbf{v}^{H} \mathbf{H}_{\mathrm{BU}} \mathbf{H}_{\mathrm{BU}}^{H} \mathbf{v}\right) .
\end{aligned}
$$

Since $\mathbf{w}_{\mathrm{T}}$ is fixed in this subsection, the work can be reduced to maximize $\phi(\mathbf{v})=\mathbf{h}_{\mathrm{BU}} \mathbf{h}_{\mathrm{BU}}^{H}+2 \operatorname{Re}\left\{\mathbf{v}^{H} \mathbf{H}_{\mathrm{BU}} \mathbf{h}_{\mathrm{BU}}^{H}\right\}+$ $\mathbf{v}^{H} \mathbf{H}_{\mathrm{BU}} \mathbf{H}_{\mathrm{BU}}^{H} \mathbf{v}$, which corresponds to the following problem

$$
\mathcal{Q}_{4}^{v}: \min _{\mathbf{v}} \mathbf{v}^{H}\left(-\mathbf{H}_{\mathrm{BU}} \mathbf{H}_{\mathrm{BU}}^{H}\right) \mathbf{v}-2 \operatorname{Re}\left\{\mathbf{v}^{H} \mathbf{H}_{\mathrm{BU}} \mathbf{h}_{\mathrm{BU}}^{H}\right\} \text { s.t.C3. }
$$

We note that the objective function in problem $\mathcal{Q}_{4}^{v}$ is concave, which makes the problem non-convex. However, it can be solved via SCA. To approximate the objective function, the following key lemma is needed.
Lemma 2 [21]: Assuming $\mathbf{Q}$ be an $N \times N$ Hermitian matrix, for any $\mathbf{x}^{(n)} \in \mathbb{C}^{N \times 1}$, one obtains that $\mathbf{x}^{H} \mathbf{Q} \mathbf{x} \leq \mathbf{x}^{H} \lambda_{1}(\mathbf{Q}) \mathbf{I} \mathbf{x}-2 \operatorname{Re}\left\{\mathbf{x}^{H}\left(\lambda_{1}(\mathbf{Q}) \mathbf{I}-\mathbf{Q}\right) \mathbf{x}^{(n)}\right\}+$ $\mathbf{x}^{(n), H}\left(\lambda_{1}(\mathbf{Q}) \mathbf{I}-\mathbf{Q}\right) \mathbf{x}^{(n)}$, where the term $\lambda_{1}(\mathbf{Q})$ denotes the maximum eigenvalues of $\mathbf{Q}$.

Proof: Please refer to [21].

Utilizing lemma 2, the upper bound of objective function in problem $\mathcal{Q}_{4}^{v}$ can be formulated as

$$
\begin{aligned}
& \mathbf{v}^{H}\left(-\mathbf{H}_{\mathrm{BU}} \mathbf{H}_{\mathrm{BU}}^{H}\right) \mathbf{v}-2 \operatorname{Re}\left\{\mathbf{v}^{H} \mathbf{H}_{\mathrm{BU}} \mathbf{h}_{\mathrm{BU}}^{H}\right\} \\
& \leq \mathbf{v}^{H} \lambda_{1}\left(-\mathbf{H}_{\mathrm{BU}} \mathbf{H}_{\mathrm{BU}}^{H}\right) \mathbf{I} \mathbf{v}-2 \operatorname{Re}\left\{\mathbf{v}^{H} \mathbf{H}_{\mathrm{BU}} \mathbf{h}_{\mathrm{BU}}^{H}\right\} \\
& -2 \operatorname{Re}\left\{\mathbf{v}^{H}\left(\lambda_{1}\left(-\mathbf{H}_{\mathrm{BU}} \mathbf{H}_{\mathrm{BU}}^{H}\right) \mathbf{I}+\mathbf{H}_{\mathrm{BU}} \mathbf{H}_{\mathrm{BU}}^{H}\right) \mathbf{v}^{(n)}\right\} \\
& +\mathbf{v}^{(n), H}\left(\lambda_{1}\left(-\mathbf{H}_{\mathrm{BU}} \mathbf{H}_{\mathrm{BU}}^{H}\right) \mathbf{I}+\mathbf{H}_{\mathrm{BU}} \mathbf{H}_{\mathrm{BU}}^{H}\right) \mathbf{v}^{(n)} .
\end{aligned}
$$

Since $\mathbf{v}^{H} \mathbf{v}=N$, the first term of (22) can be regarded as a constant. Therefore, by dropping the constant terms in (22), the majorized problem $\mathcal{Q}_{4}^{v}$ is given by

$$
\hat{\mathcal{Q}}_{4}^{v}: \max _{\mathbf{v}} \operatorname{Re}\left\{\mathbf{v}^{H}\left(\mathbf{R}_{\mathrm{BU}}+\mathbf{H}_{\mathrm{BU}} \mathbf{h}_{\mathrm{BU}}^{H}\right)\right\} \text { s.t.C3, }
$$

where $\mathbf{R}_{\mathrm{BU}}=\left(\lambda_{1}\left(-\mathbf{H}_{\mathrm{BU}} \mathbf{H}_{\mathrm{BU}}^{H}\right) \mathbf{I}+\mathbf{H}_{\mathrm{BU}} \mathbf{H}_{\mathrm{BU}}^{H}\right) \mathbf{v}^{(n)}$. According to [21], a first-order optimal closed-form solution of $\mathbf{v}$ in problem $\mathcal{Q}_{4}^{v}$ can be obtained, i.e.,

$$
\mathbf{v}^{\text {opt }}=\exp \left\{j \arg \left(\mathbf{R}_{\mathrm{BU}}+\mathbf{H}_{\mathrm{BU}} \mathbf{h}_{\mathrm{BU}}^{H}\right)\right\} .
$$

Thus, the upper bound of $\eta$ can be finally obtained as $\mathcal{U}\left(\mathbf{v}^{\mathrm{opt}}\right)=\left\|\mathbf{w}_{\mathrm{T}}\right\|^{2}\left\|\mathbf{h}_{\mathrm{BU}}+\mathbf{v}^{\mathrm{opt}, H} \mathbf{H}_{\mathrm{BU}}\right\|^{2}-\sigma_{\mathrm{U}}^{2}$. Then, problem $\mathcal{Q}_{3}^{v}$ can be equivalently expressed as

$$
\mathcal{Q}_{5}^{v}: \max _{\eta} \vartheta(\eta) \text {, s.t. } 0 \leq \eta \leq \mathcal{U}\left(\mathbf{v}^{\mathrm{opt}}\right),
$$

where $\vartheta(\eta)$ is given by

$\mathcal{Q}_{6}^{v}: \quad \vartheta(\eta)=\max _{\mathbf{v},\left\{p_{1}, p_{2}, p_{3}, p_{4}, u_{1}, u_{2}\right\} \geq 0} \frac{\left|\widetilde{\mathbf{h}}_{\mathrm{BU}} \mathbf{w}_{\mathrm{T}}\right|^{2}}{\eta+\sigma_{\mathrm{U}}{ }^{2}}$ s.t. $\overline{\mathrm{C} 1}, \mathrm{C} 3, \overline{\mathrm{C} 4}$.

To optimize $\mathbf{v}$ given $\eta$, the problem can be reformulated as

$\mathcal{Q}_{7}^{v}: \min _{\mathbf{v},\left\{p_{1}, p_{2}, p_{3}, p_{4}, u_{1}, u_{2}\right\} \geq 0} r(\mathbf{v})=-\left|\left(\mathbf{h}_{\mathrm{BU}}+\mathbf{v}^{H} \mathbf{H}_{\mathrm{BU}}\right) \mathbf{w}_{\mathrm{T}}\right|^{2}$

$$
\text { s.t. } \overline{\mathrm{C} 1}, \mathrm{C} 3, \overline{\mathrm{C}} 4 \text {. }
$$

However, problem $\mathcal{Q}_{7}^{v}$ is difficult to solve due to the objective function $r(\mathbf{v})$ and constraint C3. Therefore, we use (14) to approximate $r(\mathbf{v})$ to a linear expression, and propose a $\mathrm{P}$ CCP to relax constraint C3 by adding slack variable so that the problem $\mathcal{Q}_{7}^{v}$ can be solved [22]. Applying (14), $r(\mathbf{v})$ can be approximated as

$$
\begin{aligned}
& \widetilde{r}\left(\mathbf{v}, \mathbf{v}^{(n)}\right)=\left(\mathbf{h}_{\mathrm{BU}}+\mathbf{v}^{(n), H} \mathbf{H}_{\mathrm{BU}}\right) \mathbf{w}_{\mathrm{T}} \mathbf{w}_{\mathrm{T}}^{H}\left(\mathbf{h}_{\mathrm{BU}}^{H}+\mathbf{H}_{\mathrm{BU}}^{H} \mathbf{v}^{(n)}\right) \\
& -2 \operatorname{Re}\left\{\left(\mathbf{h}_{\mathrm{BU}}+\mathbf{v}^{(n), H} \mathbf{H}_{\mathrm{BU}}\right) \mathbf{w}_{\mathrm{T}} \mathbf{w}_{\mathrm{T}}^{H}\left(\mathbf{h}_{\mathrm{BU}}^{H}+\mathbf{H}_{\mathrm{BU}}^{H} \mathbf{v}\right)\right\},
\end{aligned}
$$

where $\mathbf{v}^{(n)}$ is optimal solution obtained at iteration $n$. Then, we introcuce two real vectors $\mathbf{b}=\left[b_{1}, b_{2}, \cdots, b_{N}\right]^{T}$ and $\mathbf{c}=$ $\left[c_{1}, c_{2}, \cdots, c_{N}\right]^{T}$, and then obtain the following constraint

$$
\overline{\mathrm{C} 3}: 1-b_{i} \leq\left|v_{i}\right|^{2} \leq 1+c_{i}, \forall i,
$$

which leads the feasible set of $\left|v_{i}\right|$ to a continuous region [17]. Note taht the first inequality in $\overline{\mathrm{C} 3}$ is still nonconvex, according to (14), it can also be approximated as 
$2 \operatorname{Re}\left\{v_{i}^{(n), *} v_{i}\right\}-\left|v_{i}^{(n)}\right|^{2} \geq 1-b_{i}$, where $v_{i}^{(n)}$ is optimal solution obtained at iteration $n$. To guarantee that the slack variables can converge to zero, we add the sum of the violations $\sum_{i=1}^{N} b_{i}+\sum_{i=1}^{N} c_{i}$ into the objective function based on the concept of P-CCP, which is penalized by a penalty parameter $\gamma$ [22]. After the aforementioned manipulations, problem $\mathcal{Q}_{7}^{v}$ can be reconstructed as

$$
\begin{aligned}
\mathcal{Q}^{v}: & \min _{\mathbf{v}} \widetilde{r}\left(\mathbf{v}, \mathbf{v}^{(n)}\right)+\gamma\left(\sum_{i=1}^{N} b_{i}+\sum_{i=1}^{N} c_{i}\right) \\
& \text { s.t. } 2 \operatorname{Re}\left\{v_{i}^{(n), *} v_{i}\right\}-\left|v_{i}^{(n)}\right|^{2} \geq 1-b_{i},\left|v_{i}\right|^{2} \leq 1+c_{i}, \forall i, \\
& \overline{\mathrm{C} 1}, \overline{\mathrm{C}} 4, p_{1}, p_{2}, p_{3}, p_{4} \geq 0, u_{1}, u_{2} \geq 0, b_{i}, c_{i} \geq 0, \forall i .
\end{aligned}
$$

Hence, for the fixed $\gamma$, the approximate first-order optimal $\mathbf{v}$ can be obtained by solving problem $\mathcal{Q}^{v}$ via SCA and CVX until convergence. In general, $\gamma$ refreshes during SCA and is bounded by $\gamma_{\max }$, which can enlarge the feasibility of problem $\mathcal{Q}^{v}$ [22]. In addition, the problem $\mathcal{Q}^{v}$ is always guaranteed to converge during SCA, where the proof can be found in [17] or [22] and thus is omitted here for brevity.

Since $\eta$ lies in $\left[0, \mathcal{U}\left(\mathbf{v}^{\text {opt }}\right)\right]$, the problem $\mathcal{Q}_{6}^{v}$ can be solved by performing uniform sampling over $\eta$, and thus an optimal $\eta^{\text {opt }}$ is obtained which can achieve the maximum value of objective function in problem $\mathcal{Q}_{6}^{v}$. Once $\eta^{\text {opt }}$ is found, the optimal $\mathbf{v}^{\text {opt }}$ of original problem $\mathcal{Q}_{1}^{v}$ is achieved. Finally, under the AO framework, the final optimal point $\left[\mathbf{w}_{\mathrm{T}}^{\mathrm{opt}}, \mathbf{v}^{\mathrm{opt}}\right]$ can be obtained by alternatively solving problem $\mathcal{Q}^{w}$ and $\mathcal{Q}^{v}$ until convergence.

\section{Convergence and Complexity Analysis}

The convergence of proposed algorithm can be guaranteed based on the following details. In particular, by refering to [17], since $\mathbf{w}_{\mathrm{T}}$ and $\mathbf{v}$ are optimized in an iterative manner by using the proposed $\mathrm{AO}$ algorithm, $R_{\mathrm{U}}\left(\mathbf{w}_{\mathrm{T}}^{(1)}, \mathbf{v}^{(1)}\right) \leq$ $R_{\mathrm{U}}\left(\mathbf{w}_{\mathrm{T}}^{(2)}, \mathbf{v}^{(2)}\right) \leq \cdots \leq R_{\mathrm{U}}\left(\mathbf{w}_{\mathrm{T}}^{(k)}, \mathbf{v}^{(k)}\right)$, where $R_{\mathrm{U}}\left(\mathbf{w}_{\mathrm{T}}^{(k)}, \mathbf{v}^{(k)}\right)$ denotes the objective value of problem $\mathcal{F}$, $\mathbf{w}_{\mathrm{T}}^{(k)}$ and $\mathbf{v}^{(k)}$ are the solutions in the iteration $k$. In addition, since $\mathbf{w}_{\mathrm{T}}$ is bounded by the constraint $\mathrm{C} 2$, and $\mathbf{v}$ is bounded by the constraint $\mathrm{C} 1, R_{\mathrm{U}}\left(\mathbf{w}_{\mathrm{T}}^{(k)}, \mathbf{v}^{(k)}\right)$ is guaranteed to converge to a limit optimal point $\left[\mathbf{w}_{\mathrm{T}}^{\mathrm{opt}}, \mathbf{v}^{\mathrm{opt}}\right]$. According to [18], the complexity of optimizing $\mathbf{w}_{\mathrm{T}}$ given $\mathbf{v}$ during each $\mathrm{SCA}$ iteration is $\mathcal{O}\left((M+2)^{2}\right)$, and that of optimizing $\mathbf{v}$ given $\mathbf{w}_{\mathrm{T}}$ with fixed $\eta$ is $\mathcal{O}\left((3 N+6)^{2}\right)$. Moreover, the complexity of solving $\eta$ via (23) and SCA is about $\mathcal{O}\left(N^{2}\right)$.

\section{Simulation Results}

In this section, numerical simulations are provided to validate the proposed algorithm. We consider a BS equipped with $M=8$ antennas and the antennas number of jammer is $L=2$. It is assumed that the BS, the user, and the IRS are located at $(0,0),(150,0)$, and $(10,5)$ in meter $(\mathrm{m})$ in a 2 -D plane, respectively. Jammer is randomly located in a circle centered at $(200,0)$ with radius of $10 \mathrm{~m}$, and eavesdropper is randomly situated in a circle centered at $(160,0)$ with radius of $5 \mathrm{~m}$. Based on the 3GPP UMi model with $3.5 \mathrm{GHz}$ carrier frequency [23], we assume that all involved channel coefficients are generated by $\mathbf{H}=\sqrt{L_{0}\left(d / d_{0}\right)^{\rho}} \mathbf{h}$, where $L_{0}=-40 \mathrm{~dB}$ denotes the path loss at reference distance $d_{0}=1 \mathrm{~m}, d$ is the link distance, $\rho$ denotes the path loss exponent, and $\mathbf{h}$ is the Rician components with Rician factors $K$ [8]. The corresponding path loss exponents and Rician factors are set as $\rho_{\mathrm{BI}}=\rho_{\mathrm{JI}}=2.2$, $\rho_{\mathrm{BU}}=\rho_{\mathrm{JU}}=\rho_{\mathrm{BE}}=\rho_{\mathrm{IU}}=\rho_{\mathrm{IE}}=3$, and $K_{\mathrm{BI}}=K_{\mathrm{JI}}=K_{\mathrm{BU}}=$ $K_{\mathrm{JU}}=K_{\mathrm{BE}}=K_{\mathrm{IU}}=K_{\mathrm{IE}}=1$, respectively, by refering to [9]. And the sampling interval of $\eta$ is set as 0.01 . Other system settings as follows: $\sigma_{\mathrm{U}}^{2}=\sigma_{\mathrm{E}}^{2}=-80 \mathrm{dBm}$ as in [9], and the jammer's beamforming is set to $\mathbf{w}_{\mathrm{J}}=\sqrt{P_{\mathrm{J}}} \frac{\mathbf{h}_{\mathrm{JU}}^{H}}{\left\|\mathbf{h}_{\mathrm{JU}}\right\|_{2}}$ as in [13], where $P_{\mathrm{J}}=30 \mathrm{dBm}$. We compare the following schemes: 1) Algorithm in [9]: the scheme only considers antieavesdropping requirement in the presence of both jammer and eavesdropper with the perfect CSI; 2) Non-IRS: under the assumption that the perfect CSI is known at the BS, we design $\mathbf{w}_{\mathrm{T}}$ by solving problem $\mathcal{Q}^{w}$ without IRS. We obtain the simulation results by averaging over 200 random channel realizations.

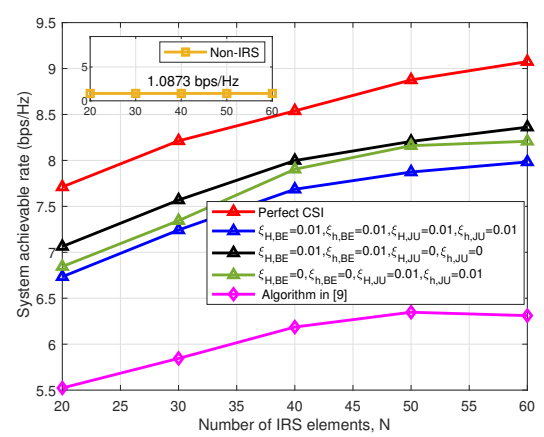

Fig. 2: System achievable rate versus $N$.

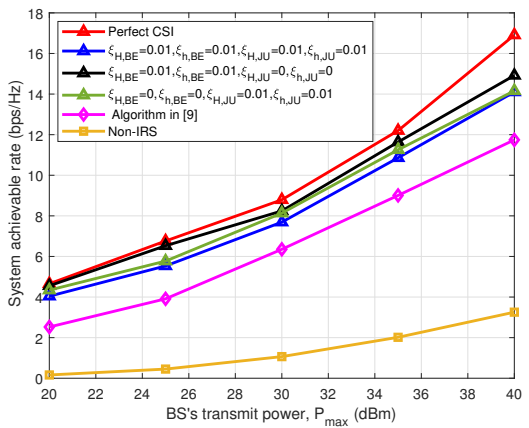

Fig. 3: System achievable rate versus $P_{\max }$.

The system achievable rate versus the BS's transmit power $P_{\max }$ is shown in Fig. 3, where $\tau=1$ and $N=50$. It is observed that as $P_{\max }$ increases, the system rate of all schemes increase despite the fact that the wiretap signal is also enhanced, and the gap between the proposed algorithms and Non-IRS scheme also increases, which indicates that the joint transmit and reflecting beamforming optimzation can effectively degrade the wiretap signal and thus achieve higher gain.

Fig. 2 shows the system achievable rate versus the number of IRS elements $N$, where $\tau=1$ and $P_{\max }=30 \mathrm{dBm}$. We find 
that all the proposed algorithms can achieve higher system rate compared to the existing approaches. In particular, the system rate of the heuristic agorithm in [9] is lower than that of proposed algorithms, which verifies the serious threat of the jammer to the system. Meanwhile, it is observed that the system rate of all schemes with IRS incresase with $N$, but the increase speed of system rate decreases with $N$. This can be explained that more RIS elements can not only exploit more degrees of freedom to enhance desired signal, but also boost the jamming signal. Hence, $N$ needs to be carefully chosen for achieving satisfactory performance. Moreover, we can also see that the system rate decreases with CSI uncertainty level, and the CSI uncertainty associated with jamming channels has a larger impact on the system rate as compared with that associated with wiretap channels.
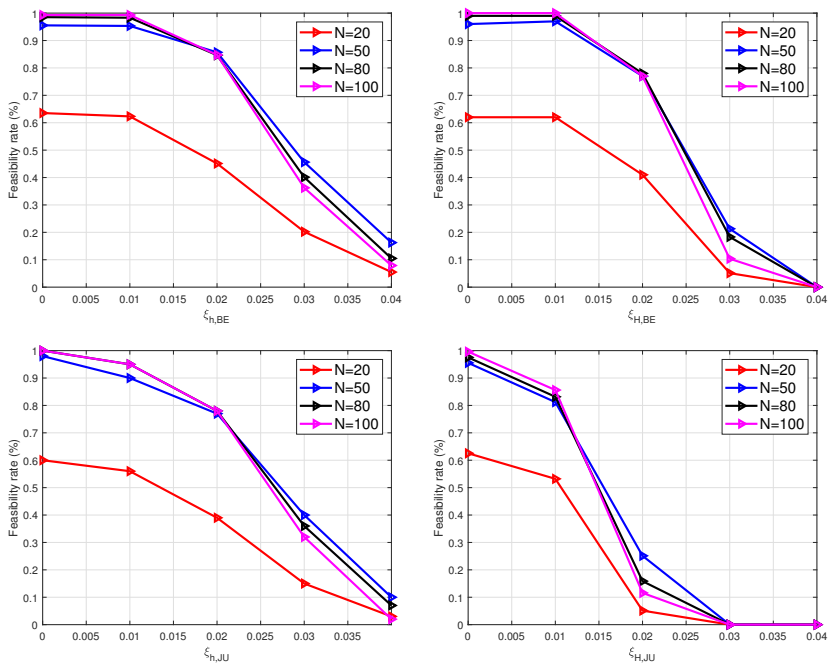

Fig. 4: Feasibility rate versus $\xi$.

Fig. 4 depicts the feasibility rate versus different $\xi$. It is observed that the feasibility rate decreases with $\xi$, and the feasibility rate of reflection channel $\left(\xi_{\mathrm{H}}\right)$ is higher than that of direct channel $\left(\xi_{\mathrm{h}}\right)$. This is because $\xi_{\mathrm{H}}$ is associated with $N$, will lead to the increase of total channel estimation errors. In addition, we can see that the feasibility rate of $N=20$ is significantly smaller than that of $N \geq 50$, and $\xi_{\mathrm{H}, \mathrm{JU}}, \xi_{\mathrm{h}, \mathrm{JU}}$ has larger negative effect on the performance as compared to $\xi_{\mathrm{H}, \mathrm{BE}}, \xi_{\mathrm{h}, \mathrm{BE}}$. This is owing to the fact that the constrain $\mathrm{C} 4$ is tighter than $\mathrm{C} 1$, and small $N$ cannot guarantee that the desired power is large enough that $\mathrm{C} 4$ can be satisfied.

\section{CONCLUSIONS}

In this paper, we have proposed a novel IRS-assisted secure transmission system against both jamming and eavesdropping attacks with imperfect CSI, and studtied the joint active transmit and passive reflecting beamforming optimization scheme to maximize the system achievable rate with transmit power and secrecy rate constraints. Specifically, the initial optimization problem was converted into a convex one by adding the auxiliary variables and utilizing General Sign-Definiteness transformation, and then SCA with P-CCP was proposed to solve the intractable problem. Numerical results confirmed that the proposed algorithm has the superior performance compared with other exsiting schemes, and the impact of channel uncertainty on the system performance was also revealed.

\section{REFERENCES}

[1] Y. Zou, J. Zhu, X. Wang, and L. Hanzo, "A survey on wireless security: Technical challenges, recent advances, and future trends," IEEE Proc., vol. 104, no. 9, pp. 1727-1765, 2016.

[2] L. Liang, W. Cheng, W. Zhang, and H. Zhang, "Mode hopping for antijamming in radio vortex wireless communications," IEEE Trans. Veh. Technol., vol. 67, no. 8, pp. 7018-7032, 2018.

[3] S. Feng and S. Haykin, "Cognitive risk control for anti-jamming V2V communications in autonomous vehicle networks," IEEE Trans. Veh. Technol., vol. 68, no. 10, pp. 9920-9934, 2019.

[4] A. Mayouche, D. Spano, C. G. Tsinos, S. Chatzinotas, and B. Ottersten, "Learning-assisted eavesdropping and symbol-level precoding countermeasures for downlink mu-miso systems," IEEE Open J. of the Commun. Society, vol. 1, pp. 535-549, 2020.

[5] S. Yan, N. Yang, I. Land, R. Malaney, and J. Yuan, "Three artificialnoise-aided secure transmission schemes in wiretap channels," IEEE Trans. Veh. Technol., vol. 67, no. 4, pp. 3669-3673, 2018.

[6] C. Pan, H. Ren, K. Wang, M. Elkashlan, A. Nallanathan, J. Wang, and L. Hanzo, "Intelligent reflecting surface aided MIMO broadcasting for simultaneous wireless information and power transfer," IEEE J. Sel. Areas Commun., vol. 38, no. 8, pp. 1719-1734, 2020.

[7] Q. Wu and R. Zhang, "Towards smart and reconfigurable environment: Intelligent reflecting surface aided wireless network," IEEE Commun. Mag., vol. 58, no. 1, pp. 106-112, 2020.

[8] —, "Intelligent reflecting surface enhanced wireless network via joint active and passive beamforming," IEEE Trans. Wireless Commun., vol. 18, no. 11, pp. 5394-5409, 2019.

[9] M. Cui, G. Zhang, and R. Zhang, "Secure wireless communication via intelligent reflecting surface," IEEE Wireless Commun. Lett., vol. 8, no. 5, pp. 1410-1414, 2019.

[10] X. Yu, D. Xu, Y. Sun, D. W. K. Ng, and R. Schober, "Robust and secure wireless communications via intelligent reflecting surfaces," IEEE J. Sel. Areas Commun., vol. 38, no. 11, pp. 2637-2652, 2020.

[11] S. Hong, C. Pan, H. Ren, K. Wang, and A. Nallanathan, "Artificial-noiseaided secure MIMO wireless communications via intelligent reflecting surface," IEEE Trans. Commun., vol. 68, no. 12, pp. 7851-7866, 2020.

[12] S. Hong, C. Pan, H. Ren, K. Wang, K. K. Chai, and A. Nallanathan, "Robust transmission design for intelligent reflecting surface-aided secure communication systems with imperfect cascaded CSI," IEEE Trans. Wireless Commun., vol. 20, no. 4, pp. 2487-2501, 2021.

[13] H. Yang, Z. Xiong, J. Zhao, D. Niyato, Q. Wu, H. V. Poor, and M. Tornatore, "Intelligent reflecting surface assisted anti-jamming communications: A fast reinforcement learning approach," IEEE Trans. Wireless Commun., pp. 1-1, 2020.

[14] G. Zhou, C. Pan, H. Ren, and A. Nallanathan, "Robust beamforming design for intelligent reflecting surface aided MISO communication systems," IEEE Wireless Commun. Lett., vol. PP, no. 99, pp. 1-1, 2020.

[15] Q. Liu, M. Li, X. Kong, and N. Zhao, "Disrupting MIMO communications with optimal jamming signal design," IEEE Trans. Wireless Commun., vol. 14, no. 10, pp. 5313-5325, 2015.

[16] J. Andrews, "Interference cancellation for cellular systems: a contemporary overview," IEEE Wireless Commun., vol. 12, no. 2, pp. 19-29, 2005.

[17] L. Dong, H.-M. Wang, and H. Xiao, "Secure cognitive radio communication via intelligent reflecting surface," IEEE Trans. Commun., pp. $1-1,2021$

[18] S. Boyd and L. Vandenberghe, "Convex optimization," Cambridge University Press, 2004.

[19] I. R. Petersen, "A stabilization algorithm for a class of uncertain linear systems." Syst. Control Lett., vol. 17, no. 2, pp. 351-357, 1987.

[20] M. Grant and B. SP, “CVX: Matlab software for disciplined convex programming," 2014.

[21] J. Song, P. Babu, and D. P. Palomar, "Optimization methods for designing sequences with low autocorrelation sidelobes," IEEE Trans. Signal Process., vol. 63, no. 15, pp. 3998-4009, 2015.

[22] T. Lipp and S. Boyd, "Variations and extension of the convexconcave procedure," Optimization Engineering, vol. 17, no. 2, pp. 263-287, 2016.

[23] 3GPP, "Technical specification group radio access network; study on 3D channel model for LTE," 2017. 